\section{A resposta da Coreia do Sul à pandemia de COVID-19: lições aprendidas e recomendações a gestores}

The response by South Korea to the COVID-19 pandemic: lessons learned and recommendations for policymakers

\author{
La respuesta de Corea del Sur a la pandemia \\ de COVID-19: lecciones aprendidas y \\ recomendaciones a gestores
}

\author{
Thais Regis Aranha Rossi 1,2 \\ Catharina Leite Matos Soares 2 \\ Gerluce Alves Silva 2 \\ Jairnilson Silva Paim 2 \\ Ligia Maria Vieira-da-Silva 2
}

\section{Resumo}

Os estudos publicados sobre a resposta da Coreia do Sul à COVID-19 apontam para distintos motivos para seu exito. Não foram identificadas revisões sobre a Coreia do Sul entre janeiro de 2020 e abril de 2021 ou que analisassem o recrudescimento da pandemia. Visando melhor sistematização sobre o seu sucesso no controle da epidemia, desenvolveu-se uma revisão integrativa para analisar a experiência daquele país no enfrentamento da pandemia de COVID-19, buscando identificar a relação entre as medidas adotadas, as características do sistema de saúde e a evolução de indicadores selecionados. Utilizaram-se distintas bases de dados, além dos boletins epidemiológicos e conferências de imprensa do Centro Sul-coreano de Prevenção e Controle de Doenças (KCDC). Adicionalmente, analisaram-se relatórios da Organização Mundial da Saúde (OMS), do Observatório Europeu de Políticas e Sistemas de Saúde. Os resultados do presente estudo permitem identificar um conjunto de lições com base na experiência sul-coreana visando o controle e manejo da doença. A resposta da Coreia do Sul foi bem-sucedida devido às ações no controle de riscos e danos, atuação sobre determinantes sociais para mitigar os efeitos socioeconômicos da crise sanitária, a experiência prévia em outras epidemias respiratórias e a coordenação nacional expressiva.

Pandemias; COVID-19; Sistemas de Saúde; Vigilância; Política de Saúde

\author{
Correspondência \\ T. R. A. Rossi \\ Universidade do Estado da Bahia. \\ Av. Silveira Martins 2555, Salvador, BA 41150-000, Brasil. \\ thais.aranha@gmail.com \\ 1 Universidade do Estado da Bahia, Salvador, Brasil. \\ 2 Instituto de Saúde Coletiva, Universidade Federal da Bahia, \\ Salvador, Brasil.
}




\section{Introdução}

A pandemia de COVID-19 representa um dos mais graves problemas sanitários em escala mundial no século XXI 1,2,3. Comparações entre as respostas de diversos países à COVID-19 mostram semelhanças e diferenças nas medidas adotadas e nos resultados obtidos. A compreensão sobre os aspectos que influenciaram o maior ou menor sucesso no controle da doença é fundamental para subsidiar os tomadores de decisão e contribuir para o planejamento voltado ao enfrentamento de futuras pandemias.

Em diversos estudos, a Coreia do Sul se apresenta como um caso bem-sucedido no controle da COVID-19 4,5,6,7,8. Nos primeiros dois meses da crise sanitária, foi reduzida a incidência, mantendo uma baixa taxa de letalidade 5 , tendo achatado rapidamente a curva epidêmica, sem o fechamento de fronteiras e estabelecimentos comerciais 6 , mesmo sendo próxima à China 8 .

Os motivos apontados para este sucesso na resposta, no entanto, são distintos. Um estudo 8 atribui o êxito à sua capacidade pré-existente, principalmente devido à revisão dos marcos legais e organizacionais após a MERS em 2015, possibilitando uma resposta rápida e eficaz pelo país durante a primeira onda da epidemia; adiciona o financiamento disponível, o sistema de governança e uma força de trabalho experiente na gestão de surtos ${ }^{8}$. Outro conjunto de estudos afirma que o sucesso do país se deve à testagem em massa da sua população, levando a identificação precoce dos casos com isolamento, ao rastreamento dos contatos e quarentena dos mesmos 9,10,11,12.

Há outro estudo que destaca que a confiabilidade dos registros de casos confirmados e suspeitos era muito alta, em torno de $95 \%$, o que facilitava o manejo das informações e as intervenções em tempo oportuno 13. Foi também citado o uso eficaz de tecnologias móveis para rastreamento, fornecimento oportuno de equipamentos de proteção individual aos trabalhadores, tratamento adequado aos infectados e conscientização da população 11,14. Segundo Nageshwaran et al. 14, o uso dessas tecnologias digitais como medida para a COVID-19 contribuiu na resposta à epidemia no país. Conforme esses autores, a vigilância digital foi efetiva para o mapeamento dos movimentos dos pacientes e fortaleceu as medidas tradicionais de saúde pública 14.

Park 12, além da testagem, atribuiu a redução na transmissão do vírus à combinação das políticas de saúde pública e socioeconômicas e infraestrutura de saúde pública associadas à transparência e informação em tempo oportuno pelas autoridades de saúde pública que constituíram uma torre de controle. Já Choi 15 ressaltou a alta coordenação nacional na produção de uma governança colaborativa, em que atores públicos e privados trabalharam pelo bem público.

Distintos autores analisam os sistemas de saúde 10,11, medidas de vigilância e estratégias adotadas 9,14. Outros se debruçam sobre a governança, medidas de vigilância e do sistema de saúde 5,16, com análises em períodos variados, compreendidos principalmente no primeiro semestre de 2020 . No entanto, não foram identificadas revisões sobre a Coreia do Sul entre janeiro de 2020 e abril de 2021 ou que analisassem o recrudescimento da pandemia.

Nesse sentido, justifica-se a importância de sistematizar as explicações apresentadas para o êxito sul-coreano. Na presente investigação buscou-se responder à seguinte pergunta: "como se deu a articulação entre o sistema de saúde e as medidas de vigilância, na resposta à pandemia de COVID-19 na Coreia do Sul?".

Com o propósito de melhor compreender o êxito no controle da epidemia na Coreia do Sul, o objetivo do presente estudo foi analisar a experiência deste país no enfrentamento da pandemia da COVID-19, buscando identificar a relação entre as medidas adotadas, as características do sistema de saúde e a evolução de indicadores selecionados.

\section{Metodologia}

Foi realizada uma revisão integrativa, adaptada da proposta de Whittemore \& Knafl 17 , tomando como objeto a resposta da Coreia do Sul à pandemia da COVID-19. Realizaram-se três buscas: a primeira entre maio e julho de 2020, a segunda no mês de novembro de 2020 e a terceira em abril de 2021, a fim de identificar os artigos que tratassem da resposta da Coreia do Sul ao aumento de novos casos confirmados de COVID-19 naquele país, face ao recrudescimento da epidemia ocorrido a partir de agosto de 2020 e abril de 2021. 
A estratégia utilizada para a primeira busca incluiu todas as partes dos artigos (título, resumo e texto completo), nas bases PubMed (https://pubmed.ncbi.nlm.nih.gov/) e Science Direct (https:// www.sciencedirect.com/). Por meio do Portal de Periódicos da Coordenação de Aperfeiçoamento de Pessoal de Nível Superior (CAPES) (https://www.periodicos.capes.gov.br/) foram acessadas as bases PubMed Central e Science Direct, o MEDLINE, One File (Gale), Directory of Open Access Journals (DOAJ), Web of Science, Wiley Online Library, Taylor \& Francis Online, BMJ Journals, Oxford Journals, SAGE Journals. Utilizaram-se descritores combinados associados aos termos "COVID-19" e "South Korea", conforme Quadro 1. Foram utilizadas as mesmas bases e estratégias de busca na segunda e terceira buscas, com restrição apenas para o título, resumo e palavras-chave. No Portal de Periódicos da CAPES, utilizou-se a restrição por título e assunto.

Adicionalmente, foram incluídos manuscritos por busca manual que constavam nas referências dos artigos selecionados, com vistas a complementar informações acerca da resposta do país à pandemia ou a possibilitar melhor compreensão sobre a organização do sistema de saúde sul-coreano.

Nas buscas, foram identificados 528 artigos com os descritores utilizados, os quais foram importados para o software EndNote (http://www.endnote.com/) e tiveram suas duplicatas removidas (Figura 1). Após a exclusão dos artigos duplicados, foram selecionados 44 para leitura na íntegra. Excluíram-se também os estudos que não focaram essa resposta ou que abordavam comparativamente países, mas sem delimitar a resposta.

Os dados foram extraídos no software Microsoft Excel (https://products.office.com/) e analisados visando à identificação das medidas adotadas pelo país estudado, a relação com a evolução da epidemia e as características do sistema de saúde. O processo de seleção dos artigos foi realizado por duas pesquisadoras independentes que entraram em consenso acerca dos trabalhos selecionados. Os procedimentos de extração de dados foram registrados em matrizes contendo o ano, título do artigo, autores, periódicos, principais achados com relação às medidas adotadas.

Utilizaram-se também os dados disponíveis na base de monitoramento da COVID-19 da Organização Mundial da Saúde (OMS), de janeiro a abril de 2021 para a Coreia do Sul (https://COVID19. who.int/table), e no Centro Sul-coreano de Prevenção e Controle de Doenças (KCDC; http://ncov. mohw.go.kr/en/). Assim como, as bases de dados do Centro Europeu de Prevenção e Controle de

\section{Quadro 1}

Bases de dados segundo estratégias de busca utilizadas.

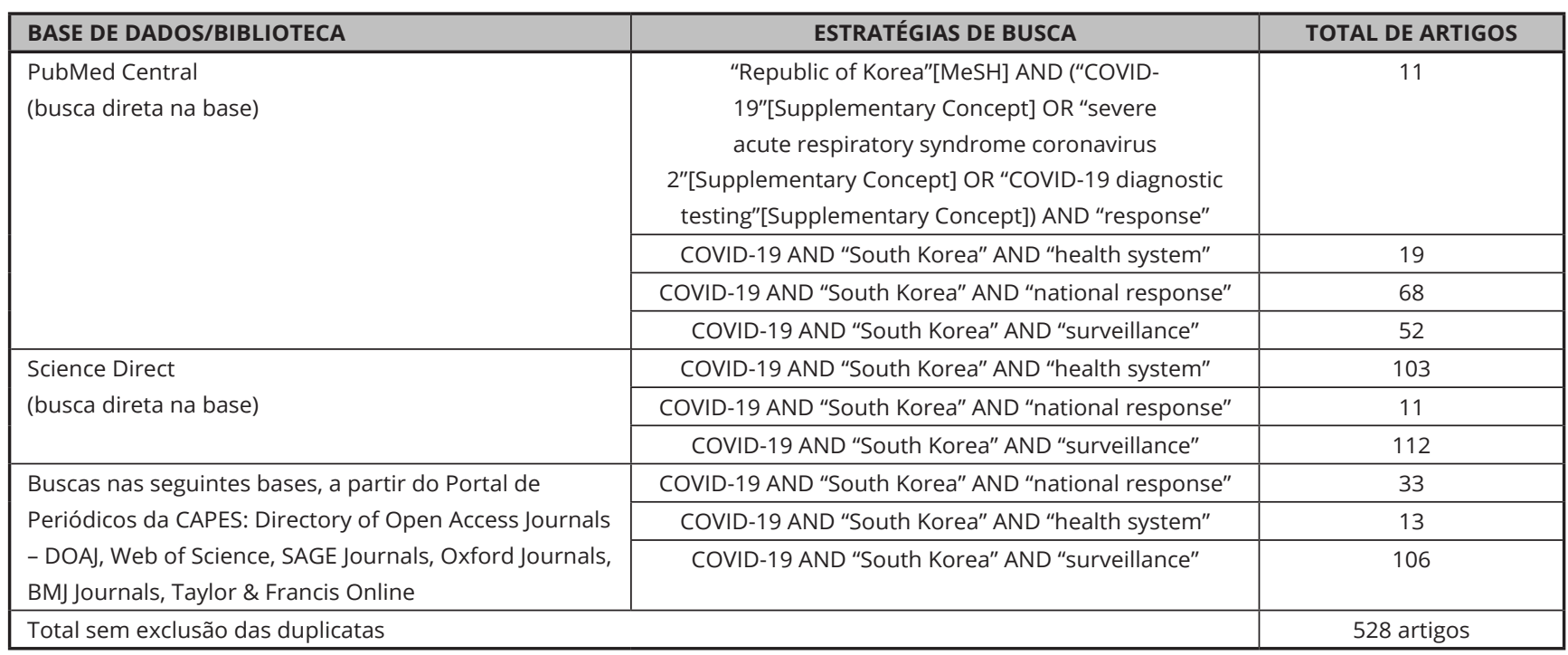


Número de artigos sobre a resposta à COVID-19 da Coreia do Sul, segundo estratégias de busca, nas bases de dados selecionadas.

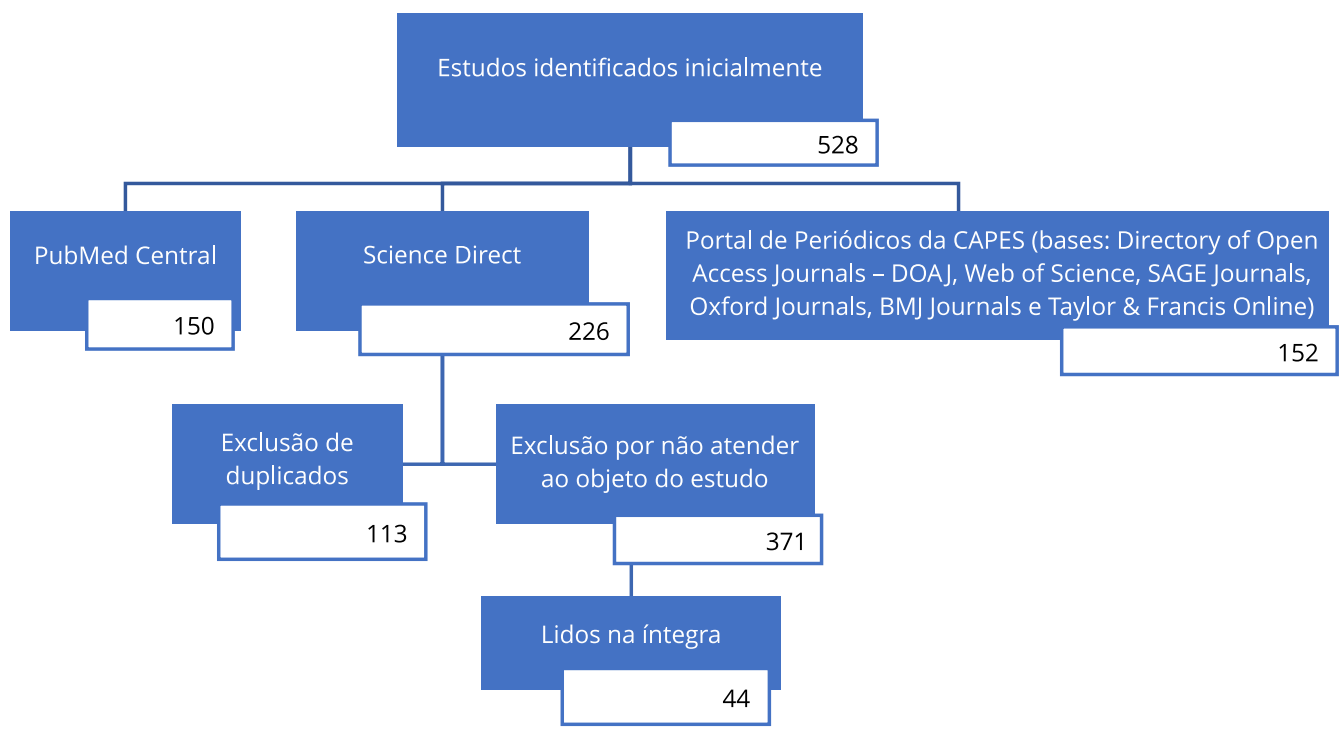

Nota: período da busca - junho de 2020 a abril de 2021.

Doenças (ECDC; https://www.ecdc.europa.eu/en/COVID-19-pandemic), e do Our World in Data (https://ourworldindata.org/). Foram analisados os relatórios da OMS, do Observatório Europeu de Políticas e Sistemas de Saúde e do KCDC, disponíveis em inglês, sobre o sistema de saúde da Coreia do Sul e sobre a reposta do país à pandemia.

A informação sobre a mobilidade em distintos locais e tipos de estabelecimento foram obtidos a partir da base de dados Google Mobility (disponível em https:/www.google.com.br/COVID19/ mobility/). Os dados foram importados em formato CSV e os gráficos elaborados no software Microsoft Excel.

Os dados foram analisados considerando três dimensões: (i) as características do país, do governo e do sistema de saúde; (ii) evolução da epidemia; e (iii) medidas adotadas em resposta à pandemia da COVID-19. Quanto às características do sistema de saúde, foram consideradas: infraestrutura, cobertura populacional na atenção à saúde, taxa de médicos por habitante, forma de prestação de serviços, financiamento e gasto com saúde, forma de organização dos serviços, histórico do sistema de saúde. Na evolução da pandemia, consideraram-se a data de ocorrência do primeiro caso, a evolução até o centésimo caso e posterior, letalidade, em relação às medidas de vigilância e controle adotadas pelo país.

\section{Resultados}

\section{Características da Coreia do Sul e seu sistema de saúde}

A Coreia do Sul é a 13a maior economia mundial, considerando o seu PIB 18. Tem uma população de mais de 52 milhões de habitantes e ocupa uma área de $100.210 \mathrm{~km}^{2}$. Trata-se de uma democracia representativa, presidencialista. Além do nível federal, tem governos regionais e locais exercidos por representantes eleitos 18 . 
O sistema de saúde é composto por dois subsistemas: (a) um baseado no seguro saúde com gerenciamento público, financiado com recursos privados, sob a responsabilidade de uma única seguradora, a National Health Insurance Corporation [Corporação Nacional de Seguro Saúde], com cobertura de $96 \%$ da população; e (b) um programa de saúde pública, denominado Medical Aid, com financiamento público por meio de impostos, voltado para os pobres, que cobre $4 \%$ da população $18,19,20$. O seguro saúde adota a compra de serviços nos prestadores privados, incluindo um componente de coparticipação dos usuários 18,19,20. Na infraestrutura, destaca-se a alta disponibilidade de leitos de UTI, em torno de 6,8/1.000 habitantes, acima da média global, concentrado no setor privado 18 .

Há registros de que esses estabelecimentos de saúde, distribuídos em distintos níveis de atenção, funcionam de modo fragmentado e desarticulado, sem a existência de uma rede resolutiva e coordenada pela atenção primária à saúde. Há múltiplas portas de entrada no sistema de saúde por meio de serviços com diferentes densidades tecnológicas 18,20,21.

Para o controle de doenças, o país apresentava estrutura vinculada ao Ministério da Saúde e Bem-Estar (MoHW), o KCDC, que lidava com a informação epidemiológica e a investigação de surtos. A gestão da saúde pública é feita de forma centralizada pelo órgão que coordena as ações de vigilância do país 18,20, dispondo de uma estrutura descentralizada, localizada nas 16 províncias e 253 centros de saúde pública locais, porém, com autonomia limitada 18.

Em 2015, o Sistema Nacional de Prevenção e Controle de Doenças Infecciosas da Coreia do Sul foi reestruturado tomando como base a avaliação não satisfatória da resposta do país à epidemia do MERS-CoV. A reforma foi desenhada a partir de proposta debatida em grande número de seminários com epidemiologistas e especialistas, além de audiências públicas.

Essa reorganização resultou na construção de um sistema de resposta inicial para conter surtos de doenças infecciosas de forma oportuna, com a criação no KCDC de um Centro de Operação de Emergências para monitorar, em tempo real, informações de doenças infecciosas e responder imediatamente aos surtos detectados. A reforma também estabeleceu um sistema especializado de diagnóstico e tratamento junto às instalações de quarentena existentes, para detecção e prevenção de surtos de doenças infecciosas emergentes, além da criação de sistema de triagem nas unidades de emergência. Houve ainda a ampliação da infraestrutura para controle da infecção no ambiente hospitalar, assim como a revisão dos modelos de gestão/governança para doenças infecciosas emergentes.

Em setembro de 2020, o KCDC foi transformado em uma agência governamental independente nomeada Agência Sul-coreana de Prevenção e Controle de Doenças (KDCA), com a incorporação do Instituto Nacional de Saúde (NIH), Instituto Nacional de Doenças Infecciosas (NIID), Centros Regionais de Prevenção e Controle de Doenças (RCDCs), Hospitais Nacionais de Tuberculoses e Estações Nacionais de Quarentena. A ampliação de sua autonomia e equipe possibilitou a essa agência mais autoridade e responsabilidade na coordenação da resposta nacional às doenças infeciosas 22 .

\section{Resposta à epidemia de COVID-19}

O governo sul-coreano incentivou o MoHW a adotar uma resposta nacional baseada em dados e na ciência 23. No início da pandemia, em março/2020, Ha 24 defendeu que a resposta deveria ser baseada nos riscos. O Secretário-Chefe do Presidente, responsável pela governança de todos os setores, era um pesquisador de sistemas de saúde, o que favoreceu a coordenação entre o escritório do PrimeiroMinistro, o Ministério de Assistência Social e o KCDC, permitindo uma resposta rápida e baseada em informações epidemiológicas e na realocação de recursos. O MoHW trabalhou junto aos governos provinciais e metropolitanos.

O protocolo de resposta ativado pelo governo buscou assegurar uma atuação coordenada e intersetorial 25, com a participação da sociedade e sob forte liderança nacional 5 . Cabe registrar que, desde 2016, o KCDC estabeleceu um sistema de avaliação de risco 26,27.

A resposta à pandemia no país foi desencadeada a partir do Plano Nacional de Gestão das Doenças Infecciosas e, com base nele, os governos locais elaboraram seus planos de execução de forma coordenada com o nível nacional 16 .

O primeiro nível de alerta foi acionado em 3 de janeiro de 2020, após a notificação dos primeiros casos de COVID-19 na China. Já o segundo nível de alerta foi ativado em 20 de janeiro de 2020, quando foi identificado o primeiro caso na Coreia do Sul 28,29. Esse primeiro caso era uma mulher vinda 
da cidade de Wuhan (China) 30 e foi identificado no aeroporto. Em 27 de janeiro de 2020, o terceiro nível de alerta foi ativado devido ao aumento dos casos. Os casos analisados entre 20 de janeiro e 10 de fevereiro de 2020 mostram que a infecção ocorreu principalmente em Wuhan, mas também em Guangdong (China), Singapura, Japão e Tailândia 30. Em 20 de fevereiro, a Coreia do Sul atingiu o centésimo caso positivo da doença e notificou a primeira morte por COVID-19 31 (Quadro 2). A incidência apresentou brusca elevação até o final do mês de fevereiro 32 (Tabela 1; Figura 2; Quadro 2).

\section{Quadro 2}

Principais medidas adotadas pela Coreia do Sul para conter a epidemia de COVID-19, segundo data (janeiro de 2020 a abril de 2021 ).

\begin{tabular}{|c|c|}
\hline PERÍODO & MEDIDAS ADOTADAS \\
\hline \multirow[t]{4}{*}{ Janeiro/2020 } & 3 de janeiro - Primeiro nível de alerta. \\
\hline & $\begin{array}{l}\text { Proibição de entrada dos oriundos da cidade de Wuhan e província de Hubei (China) e fortalecimento de medidas de } \\
\text { quarentena dos que chegavam no país. }\end{array}$ \\
\hline & 20 de janeiro - Segundo nível de alerta ativado. \\
\hline & 27 de janeiro - Terceiro nível de alerta ativado. \\
\hline Fevereiro/2020 & 23 de fevereiro - Quarto nível de alerta ativado. \\
\hline \multirow[t]{3}{*}{ Março/2020 } & 2 de março - Adiamento do início das aulas em escolas e jardins de infância de 2 março para 8 de abril. \\
\hline & $\begin{array}{l}19 \text { de março - Procedimentos especiais de quarentena para os que chegavam ao país, incluindo checagem de } \\
\text { temperatura, preenchimento de declaração sobre saúde nos aeroportos e recomendação de uso de um aplicativo de } \\
\text { celular para informar mudanças no estado de saúde. }\end{array}$ \\
\hline & $\begin{array}{l}22 \text { de março - Início da campanha de comunicação por quatro semanas (p.ex.: fique em casa e evite aglomerações), } \\
\text { estímulo às pessoas para evitarem eventos religiosos, esportes em locais fechados e equipamentos de entretenimento, } \\
\text { todos que chegavam para uma estadia longa eram obrigados a fazer um teste para COVID-19 e uma autoquarentena } \\
\text { (14 dias) em domicílio ou em instalações designadas pelo governo. }\end{array}$ \\
\hline \multirow[t]{7}{*}{ Abril/2020 } & $\begin{array}{l}\text { 1o de abril - Ampliação da política de isolamento obrigatório para todos os que chegavam no país, } \\
\text { inclusive para estadias curtas. }\end{array}$ \\
\hline & $\begin{array}{l}5 \text { de abril - Reforço de penalidade para os que violassem as medidas de isolamento com um ano de } \\
\text { prisão ou USD } 8.000 \text { em multas. }\end{array}$ \\
\hline & $\begin{array}{l}\text { A partir de } 9 \text { de abril, inicia-se gradualmente o novo ano acadêmico com aulas online, primeiro com os alunos de maior } \\
\text { idade (middle e high school) e depois o ensino elementar. }\end{array}$ \\
\hline & $\begin{array}{l}13 \text { de abril - Recomendação de que todos os coreanos e viajantes estrangeiros oriundos dos Estados Unidos fossem } \\
\text { testados nos primeiros três dias, independente dos sintomas. }\end{array}$ \\
\hline & $\begin{array}{l}20 \text { de abril - Início da fase de flexibilização de medidas de distanciamento físico, enquanto a campanha foi } \\
\text { estendida até } 5 \text { de maio. }\end{array}$ \\
\hline & $\begin{array}{l}20 \text { de abril - Permissão de encontros religiosos e algumas atividades sociais desde que fossem mantidos } \\
\text { os protocolos de higiene. }\end{array}$ \\
\hline & $\begin{array}{l}22 \text { de abril - Divulgação dos protocolos para que indivíduos e comunidades equilibrassem } \\
\text { atividades diárias e de quarentena. }\end{array}$ \\
\hline \multirow[t]{3}{*}{ Maio/2020 } & Início de maio - Começo da temporada dos jogos de basquete profissional sem público. \\
\hline & $\begin{array}{l}6 \text { de maio - Liberação de medidas de distanciamento e mudança para "quarentena da vida cotidiana" e encerramento } \\
\text { de seis semanas de campanha de distanciamento social forte, com mudança para o chamado esquema de quarentena } \\
\text { da vida cotidiana, “5 Regras Básicas”: (1) fique em casa por 3-4 dias se você ficar doente; (2) mantenha uma distância } \\
\text { de dois braços entre você e outras pessoas; (3) lave as mãos por } 30 \text { segundos. Tosse/espirro na manga; (4) ventile seu } \\
\text { espaço pelo menos duas vezes ao dia e desinfete-o regularmente; e (5) mantenha contato com a família e amigos, mas } \\
\text { com distanciamento físico. Orientação aos idosos de manutenção dos medicamentos para doenças } \\
\text { crônicas e contato com os centros de saúde pública em casos de sintomas. No nível comunitário, alertava-se para } \\
\text { importância da atuação de todos. }\end{array}$ \\
\hline & $\begin{array}{l}\text { Em } 6 \text { de maio, as escolas abriram gradualmente, no entanto, as universidades que em } 9 \text { de março iniciaram com aulas } \\
\text { online, estenderam essa modalidade de ensino até o fim do semestre de primavera. }\end{array}$ \\
\hline
\end{tabular}

(continua) 
Quadro 2 (continuação)

\begin{tabular}{|c|c|}
\hline PERÍODO & MEDIDAS ADOTADAS \\
\hline Julho/2020 & $\begin{array}{l}26 \text { de julho - Paulatinamente permissão para os torcedores frequentarem os eventos esportivos } \\
\text { como beisebol e futebol. }\end{array}$ \\
\hline \multirow[t]{2}{*}{ Agosto/2020 } & 23 de agosto - Medida de distanciamento social nível 2 é aplicada em todo o país. \\
\hline & 30 de agosto - Medida de distanciamento social “aprimorada” de nível 2 é aplicada para Seul, área metropolitana. \\
\hline \multirow[t]{2}{*}{ Setembro/2020 } & $\begin{array}{l}14 \text { de setembro - Distanciamento social nível 2, KCDC implementa reforço de medidas, tais como contratação e } \\
\text { qualificação de mais oficiais de inteligência epidemiológica, esforços de pesquisa e desenvolvimento relacionados a } \\
\text { doenças infecciosas, incluindo ensaios clínicos e apoio ao desenvolvimento de vacinas, por meio do recém-formado } \\
\text { Instituto Nacional de Doenças Infecciosas (NIID); apoio aos esforços de resposta local em coordenação mais próxima } \\
\text { por meio dos } 5 \text { Centros Regionais para Controle e Prevenção de Doenças, reforço do monitoramento e análise para } \\
\text { resposta rápida, por meio do call center 1339; Reforço do suprimento e gerenciamento de vacina. }\end{array}$ \\
\hline & $\begin{array}{l}25 \text { de setembro - Recomendações para o feriado da Colheita no outono: permanência com os familiares mais próximos } \\
\text { em casa; manutenção da distância de } 2 \text { metros, uso de máscara no domicílio e ao ar livre, se não houver possibilidade } \\
\text { de manutenção dos } 2 \text { metros de distância, dar preferência a locais abertos e ventilados, sem aglomerações. Atividades } \\
\text { físicas em parques de bairros, trilhas próximas e parques recreativos. Atividades culturais, com reservas prévias e uso } \\
\text { das programações de TV. Para a população idosa, pais e avós, preferir saudações por videochamadas. }\end{array}$ \\
\hline \multirow[t]{2}{*}{ Outubro/2020 } & 19 de outubro - Divulgação de informações sobre vigilância da gripe e calendário de vacinação para influenza. \\
\hline & $\begin{array}{l}27 \text { de outubro - Orientação de distanciamento social para segurança no Halloween. Evitar eventos, sobretudo se } \\
\text { tiver febre ou sintomas respiratórios; incentivo às reuniões não presenciais e sem contato; evitar clubes, bares, pubs, } \\
\text { restaurantes, cafés e locais de diversão noturna, se possível. Para compromissos inevitáveis, recomendação de uso das } \\
\text { medidas de segurança (evitar horários de movimento, compartilhamento de fantasias, observância de sintomas, uso } \\
\text { de máscaras, distanciamento de } 2 \text { metros, higiene das mãos, evitar contato físico, não compartilhamento de copos e } \\
\text { utensílios, medida de temperatura, desinfeção dos espaços físicos). Em caso de participação de encontro grupal, caso } \\
\text { testagem positiva, informar aos outros participantes para que possam fazer o teste. }\end{array}$ \\
\hline \multirow[t]{3}{*}{ Novembro/2020 } & 11 de novembro - Disposição sobre as medidas de distanciamento dos níveis 1 a 3. \\
\hline & $\begin{array}{l}19 \text { de novembro - Elevação dos níveis de distanciamento social para 1,5m, em Seul, na Província de Gyeonggi e na } \\
\text { cidade de Icheon. }\end{array}$ \\
\hline & $\begin{array}{l}24 \text { de novembro - Elevação do nível de distanciamento social para } 2 \mathrm{~m} \text {, nas áreas de Seul, Incheon, Gyeonggi e nível } \\
\text { 1,5m para Honam e região (Gwangju, Jeonbuk e Jeonnam): home office para 1/3 da força de trabalho, rodízio para } \\
\text { os horário de trabalho e almoço, uso de máscara em todos os espaços internos, suspensão de todas as viagens de } \\
\text { negócios não essenciais, cancelamento de todas as reuniões não essenciais na modalidade presencial. }\end{array}$ \\
\hline \multirow[t]{4}{*}{ Dezembro/2020 } & 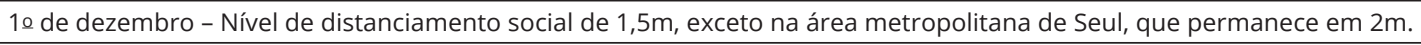 \\
\hline & 7 de dezembro - Revisão dos critérios para encerrar o isolamento para casos de COVID-19. \\
\hline & $\begin{array}{l}8 \text { de dezembro - Distanciamento social de 2,5m, para a área metropolitana de Seul; reforço ao home office, ensino } \\
\text { à distância, proibição de aulas particulares presenciais, restrição de deslocamento para outras regiões. Restrição de } \\
\text { funcionamento de bares e restaurantes e outras instalações até as } 21 \text { horas; limitação de eventos a } 50 \text { ou menos } \\
\text { participantes, acomodações não permitidas para festas; restrição de reuniões e atividade física em ambientes fechados; } \\
\text { comida para viagem apenas em cafés; fechamento de saunas; proibição de eventos religiosos. Nas demais áreas do } \\
\text { país, destinação de medidas mais restritivas, nível de distanciamento social 2: Entrega de restaurantes até as } 21 \text { horas, } \\
\text { fechamento de clubes e pubs noturnos, fechamento de salas de canto e academias às } 21 \text { horas; uso obrigatório de } \\
\text { máscara, eventos com limite de } 100 \text { participantes, limitação de alunos presenciais nas escolas, limitação de participantes } \\
\text { presenciais em eventos religiosos regulares. }\end{array}$ \\
\hline & $\begin{array}{l}12 \text { de dezembro - Distanciamento social nível } 1 \text { - Solicitação para a população quanto a vigilância. Na área } \\
\text { metropolitana, o surgimento de aglomerados locais, manutenção de algumas medidas do nível 2, tais como: uso } \\
\text { obrigatório de máscaras em locais de controle de infecção obrigatório e medidas de prevenção para instalações de alto } \\
\text { risco, locais com risco de exposição a um grande número de pessoas como transporte público e locais de protesto, bem } \\
\text { como instalações com grupos populacionais vulneráveis, como hospitais, cuidados domiciliares, independentemente do } \\
\text { nível de distanciamento em vigor. Uso de multas caso haja uso inadequado de máscaras. }\end{array}$ \\
\hline
\end{tabular}

(continua) 
Quadro 2 (continuação)

\begin{tabular}{|c|c|}
\hline PERÍODO & MEDIDAS ADOTADAS \\
\hline Dezembro/2020 & $\begin{array}{l}21 \text { de dezembro - Emissão de medidas especiais de prevenção de infecções para as férias de inverno, incluem: testagem } \\
\text { em Hospitais de convalescença, hospitais psiquiátricos e ambulatórios; teste diagnóstico obrigatório a cada } 1-2 \\
\text { semanas ou teste rápido de antígeno } 1 \text {-2 vezes por semana); restrição de entrada de pessoal não autorizado; a equipe } \\
\text { não tem permissão para participar de reuniões sociais privadas. Nas instalações religiosas, manutenção de medidas } \\
\text { nível 2,5m aplicadas em todo o país, com missas regulares não presenciais. Reuniões privadas, limitadas a } 5 \text { pessoas, } \\
\text { exceto membros permanentes, não permitido em restaurantes. Proibição de aluguel de salões de festa, medição de } \\
\text { temperaturas em estabelecimentos de grande circulação. Ocupação máxima em resorts 50\% e proibição de estância } \\
\text { de esqui, resort de trenó, pistas de patinação no gelo e outras instalações para esportes de inverno. Fechamento de } \\
\text { destinos populares para viagens de ano novo, incluindo locais do pôr do sol, nascer do sol e parques nacionais. }\end{array}$ \\
\hline Janeiro/2021 a & 22 de fevereiro - Início da vacinação contra COVID-19. \\
\hline Abril/2021 & $\begin{array}{l}20 \text { de abril - Após } 87 \text { dias, os casos diários novamente ultrapassam os } 600 \text {, alimentando as preocupações com uma } \\
\text { quarta onda. O KDCA apontou que a quarta onda de casos deve ser evitada durante a fase de vacinação dos idosos. } \\
\text { Medidas de prevenção reforçadas e pactuações em distintos níveis governamentais. }\end{array}$ \\
\hline
\end{tabular}

KCDC: Centro Sul-coreano de Prevenção e Controle de Doenças; KDCA: Agência Sul-coreana de Prevenção e Controle de Doenças.

Fonte: KCDC/KCDA (http://www.kdca.go.kr/, acessado em 30/Abr/2021).

O alerta no país alcançou o nível mais alto (nível 4), principalmente devido ao surto massivo em Daegu, em 23 de fevereiro de 2020 (Figuras 2 e 3). O governo acionou protocolos de resposta nacional com vistas à articulação entre a sociedade e distintos níveis de governo para conter a pandemia 5 e instalou a Central de Contramedidas de Segurança e Desastres, chefiada pelo Primeiro-Ministro. Em 21 de março, 56,8\% dos casos, ou seja, 5.051 dos 8.897 casos confirmados em todo o país estavam vinculados ao 31o paciente associado à Igreja Sincheonji de Jesus 5. Este foi o maior cluster associado à disseminação da doença com epicentro na cidade de Daegu 32 , seguido pelo do hospital Chung do Daenam na mesma cidade 33. O fato da contaminação em massa na igreja, responsável por grande disseminação do vírus, foi chamado de "cisne negro" 34 . O aumento do Rt no mês de abril esteve relacionado com o crescimento de grupos de transmissão local na Região Metropolitana de Seul (Seul, cidade de Incheon e província de Gyeonggi) 35. A distribuição de casos recuperados foi maior entre as mulheres 36 .

A Coreia do Sul desenvolveu uma abordagem com foco em duas linhas: resposta clínica para gerenciar os casos, e a resposta da saúde pública, a fim de prevenir e detectar novas infecções 5 . Quanto ao gerenciamento dos casos, as ações no sistema de saúde foram separadas em dois subsistemas, "COVID-19" e "não COVID-19", com vistas à garantia da continuidade do cuidado das demais necessidades da população. A governança e a coordenação nacional foram relevantes para a oferta e fluxo de entrega de suprimentos e na organização de sistema de referência, com encaminhamento dos pacientes entre os níveis de atenção e nas fronteiras das cidades metropolitanas ou territórios provinciais, mesmo na ausência de uma estrutura legal clara.

Na atenção aos casos de COVID-19, a baixa taxa de letalidade apresentada pelo país atribui-se à capacidade de reduzir a taxa de infecção entre populações de alto risco, por meio de detecção e isolamento precoce dos casos 37 , o que impediu a sobrecarga dos hospitais, disponibilizando-os para o cuidado dos casos graves 5 .

Ampliou-se, ainda, a infraestrutura para o enfrentamento de COVID-19 no país com: (i) implantação de Centros de Triagem no nível distrital para avaliar indivíduos com febre ou sintomas respiratórios; (ii) organização de Unidades de Terapia Intensiva com pressão negativa nos hospitais universitários e credenciados às mesmas; (iii) definição de hospitais e alocação de especialistas em doenças respiratórias para tratamento de pacientes não críticos; (iv) implantação de leitos clínicos em espaços "não hospitalares" (hospitais de campanha) para tratamento de casos leves, com qualificação 
Tabela 1

Novos casos, total acumulado de casos, novas mortes, total de mortes, letalidade, mortes/milhão, testes por caso e total de testes por COVID-19, segundo data de registro. Coreia do Sul, janeiro de 2020 a abril de 2021.

\begin{tabular}{|c|c|c|c|c|c|c|c|c|}
\hline Data & Novos casos & $\begin{array}{l}\text { Total de casos, } \\
\text { acumulados a }\end{array}$ & $\begin{array}{l}\text { Novas } \\
\text { mortes }\end{array}$ & $\begin{array}{l}\text { Total de } \\
\text { mortes b }\end{array}$ & $\begin{array}{l}\text { Letalidade } \\
\text { (\%) [b/a] }\end{array}$ & Mortes * & $\begin{array}{c}\text { Testes por } \\
\text { caso [c/b] }\end{array}$ & $\begin{array}{c}\text { Total de } \\
\text { testes, } \\
\text { acumulados c }\end{array}$ \\
\hline 19/Jan/2020 & 5 & 5 & 0 & 0 & 0,00 & 0,00 & 0,00 & 0 \\
\hline 30/Jan/2020 & 2 & 10 & 0 & 0 & 0,00 & 0,00 & 24,40 & 244 \\
\hline 15/Fev/2020 & 0 & 32 & 0 & 0 & 0,00 & 0,00 & 241,69 & 7.734 \\
\hline 29/Fev/2020 & 813 & 3.154 & 4 & 17 & 0,50 & 0,31 & 29,82 & 94.055 \\
\hline 15/Mar/2020 & 76 & 8.166 & 3 & 75 & 0,90 & 1,46 & 32,84 & 268.212 \\
\hline 31/Mar/2020 & 125 & 9.786 & 4 & 162 & 1,70 & 3,16 & 41,95 & 410.564 \\
\hline 15/Abr/2020 & 27 & 10.591 & 3 & 225 & 2,10 & 4,33 & 50,47 & 534.552 \\
\hline 30/Abr/2020 & 4 & 10.765 & 1 & 247 & 2,30 & 4,84 & 57,58 & 619.881 \\
\hline 15/Mai/2020 & 27 & 11.018 & 0 & 260 & 2,40 & 5,11 & 65,96 & 726.747 \\
\hline 31/Mai/2020 & 27 & 11.468 & 1 & 270 & 2,40 & 5,29 & 79,42 & 910.822 \\
\hline 15/Jun/2020 & 36 & 12.121 & 0 & 277 & 2,30 & 5,42 & 91,22 & 1.105 .719 \\
\hline 30/Jun/2020 & 43 & 12.800 & 0 & 282 & 2,20 & 5,50 & 99,51 & 1.273 .766 \\
\hline 15/Jul/2020 & 39 & 13.551 & 0 & 289 & 2,10 & 5,68 & 105,62 & 1.431 .316 \\
\hline 31/Jul/2020 & 36 & 14.305 & 1 & 301 & 2,10 & 5,87 & 109,32 & 1.563 .796 \\
\hline 15/Ago/2020 & 166 & 15.039 & 0 & 305 & 2,03 & 5,95 & 111,40 & 1.675 .296 \\
\hline 30/Ago/2020 & 299 & 19.699 & 2 & 323 & 1,64 & 6,32 & 97,68 & 1.924 .170 \\
\hline $15 /$ Set/2020 & 106 & 22.391 & 4 & 367 & 1,64 & 7,16 & 96,67 & 2.164 .578 \\
\hline $30 /$ Set/2020 & 113 & 23.812 & 6 & 413 & 1,73 & 8,09 & 97,56 & 2.322 .999 \\
\hline 15/Out/2020 & 110 & 24.998 & 1 & 436 & 1,74 & 8,60 & 98,04 & 2.450 .739 \\
\hline $30 /$ Out/2020 & 113 & 26.384 & 1 & 463 & 1,75 & 9,05 & 99,01 & 2.612 .231 \\
\hline 15/Nov/2020 & 208 & 28.546 & 1 & 493 & 1,73 & 9,63 & 97,63 & 2.786 .878 \\
\hline $30 /$ Nov/2020 & 439 & 34.201 & 3 & 526 & 1,53 & 10,26 & 89,50 & 3.061 .172 \\
\hline 15/Dez/2020 & 880 & 44.364 & 13 & 600 & 1,35 & 11,94 & 77,56 & 3.441 .220 \\
\hline 30/Dez/2020 & 1.050 & 59.773 & 20 & 879 & 1,47 & 17,55 & 69,58 & 4.159 .522 \\
\hline 15/Jan/2021 & 513 & 71.241 & 22 & 1.217 & 1,70 & 24,11 & 69,88 & 4.978 .075 \\
\hline 30/Jan/2021 & 458 & 77.850 & 15 & 1.414 & 1,81 & 27,70 & 72,15 & 5.616 .530 \\
\hline 15/Fev/2021 & 344 & 83.869 & 5 & 1.527 & 1,82 & 29,92 & 73,48 & 6.162 .860 \\
\hline 28/Fev/2021 & 356 & 89.676 & 8 & 1.603 & 1,79 & 31,30 & 74,14 & 6.649 .006 \\
\hline 15/Mar/2021 & 382 & 96.017 & 6 & 1.675 & 1,74 & 32,73 & 73,79 & 7.084 .940 \\
\hline 30/Mar/2021 & 447 & 102.582 & 3 & 1.729 & 1,69 & 33,76 & 74,71 & 7.663 .999 \\
\hline 15/Abr/2021 & 698 & 112.117 & 6 & 1.788 & 1,59 & 34,91 & 73,95 & 8.291 .126 \\
\hline
\end{tabular}

Fonte: Organização Mundial da Saúde 31; Our World in Data 65; Agência Sul-coreana de Prevenção e Controle de Doenças (http://www.kdca.go.kr/, acessado em 30/Abr/2021).

* Por milhão de habitantes.

dos profissionais de saúde; (v) definição da governança e coordenação nacional; e (vi) destinação de ações e insumos para a proteção dos trabalhadores de saúde.

As tecnologias de comunicação social foram usadas a fim de estimular a população na manutenção do distanciamento e do trabalho em casa 38, e o país adotou distintas medidas nessa perspectiva, variando com a situação epidemiológica ${ }^{19}$, dentre elas o cancelamento de eventos públicos, a proibição/restrição de viagens e o fechamento de escolas e universidades, as quais foram implementadas de forma progressiva (Figuras 2 e 3 ).

Com base nos procedimentos-padrão para comunicação de risco em emergências de saúde pública, implementados no país em 2017 16, desde o início da pandemia, o KCDC publicava diariamente no seu website as informações sobre a evolução da pandemia, atualizações nas estratégias, e revisão 
Figura 2

Casos e óbitos de COVID-19 notificados nas últimas 24 horas segundo data do registro e medidas adotadas. Coreia do Sul, 21 de janeiro de 2020 a 21 de janeiro de 2021.

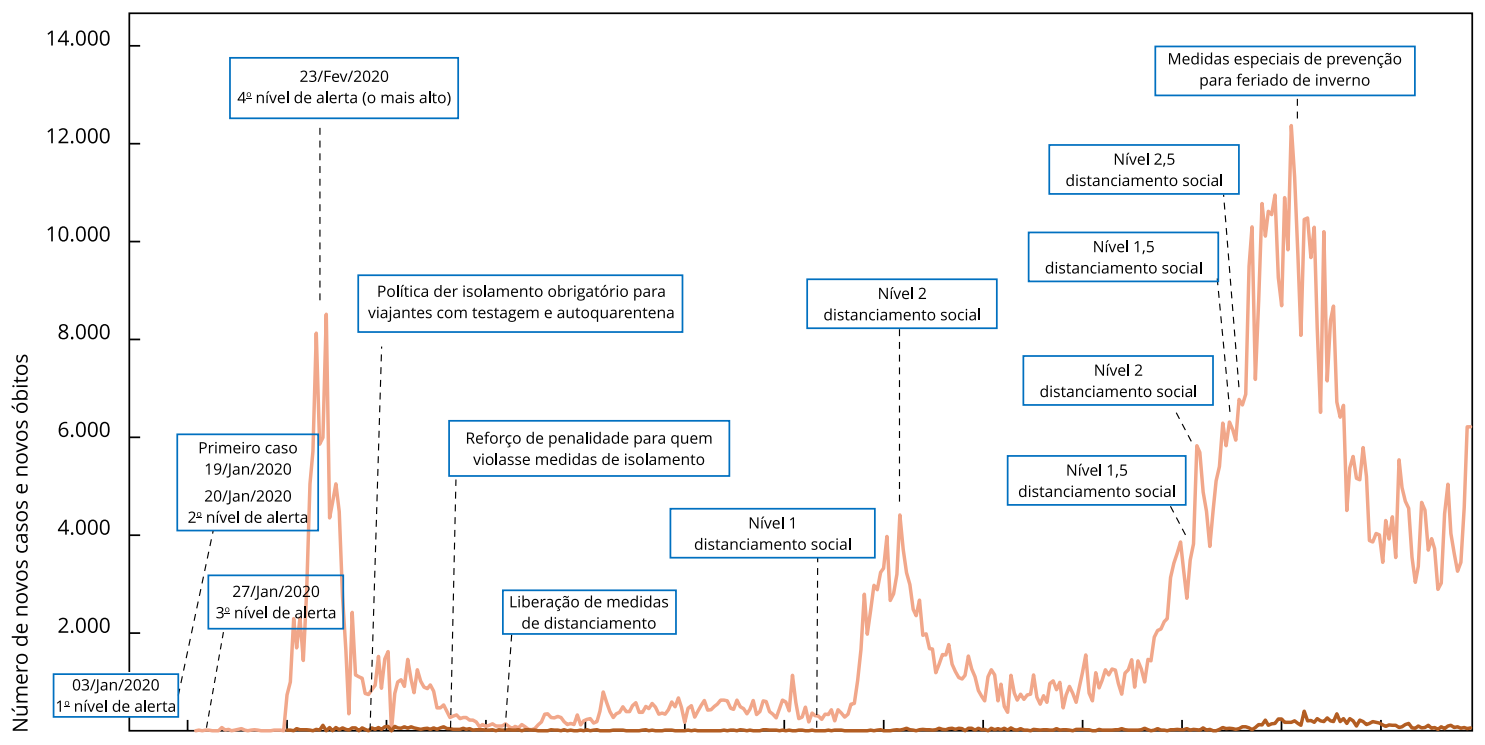

21/Jan/2020 21/Fev/2020 21/Mar/2020 21/Abr/2020 21/Mai/2020 21/Jun/2020 21/Jul/2020 21/Ago/2020 21/Set/2020 21/Out/2020 21/Nov/2020 21/Dez/2020 21/Jan/2021

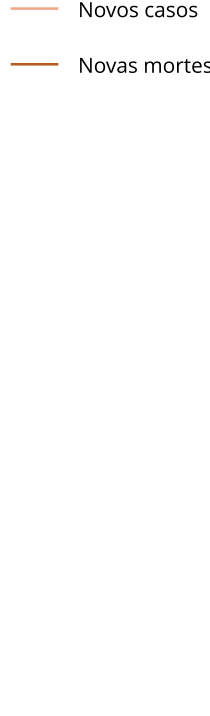

Data de notificação

Fonte: Agência Sul-coreana de Prevenção e Controle de Doenças (http://www.kdca.go.kr/, acessado em 30/Abr/2021).

dos regulamentos e das medidas em consonância com a evolução de epidemia no país 39 . As práticas de prevenção eram continuamente divulgadas pelo governo 29 como as práticas preventivas e de distanciamento físico adotadas desde o início da pandemia 40. Citam-se também as políticas para uso de máscaras faciais 41. Associações médicas manifestaram-se publicamente em apoio ao KCDC 42. Eram enviadas mensagens de alerta para os celulares dos cidadãos pelas autoridades locais, com os novos casos confirmados e suas trajetórias recentes se disponíveis. O sistema utilizado já era adotado pelo Ministério do Interior e Segurança para informar sobre desastres 39.

Os protocolos de isolamento adotados seguiram as recomendações do Regulamento Sanitário Internacional, não implicando o fechamento das fronteiras nacionais 19. Instituiu-se um programa de autoquarentena e rastreamento de contatos para todos os viajantes ${ }^{43}$, com o uso obrigatório de um aplicativo de celular 23. Indivíduos identificados como contatos de casos confirmados ou suspeitos eram colocados em quarentena domiciliar ou, quando não era possível, em centros de apoio públicos sem cobranças de taxas 5 . Ainda houve, em situações específicas, parcerias com o setor privado, visando ampliação da infraestrutura de atenção à saúde.

Em Daegu, foi operada uma rede composta pelo Grupo Consultivo de Resposta à Emergências, Associação Médica de Daegu e três grupos de suporte que atuaram de forma coordenada no controle da epidemia de COVID-19 16. No caso de Hanam, a Associação Médica, o Centro de Saúde Pública, os governos centrais e provinciais associaram-se para triar, testar e informar a população, por meio do compartilhamento da infraestrutura pública e privada, assim como a força de trabalho 44 .

No que diz respeito à vigilância de casos, a testagem ampla foi adotada como estratégia fundamental para identificar casos e rastrear contatos 39,45,46,47. A Coreia do Sul estabeleceu sua capacidade de testagem com três intervenções: parcerias público-privadas para o desenvolvimento de tecnologias de testagem que incluíram o RT-PCR, padrão-ouro para a COVID-19; aprovação do uso dos testes para casos suspeitos; e rápido desenvolvimento desses testes pelo país por meio de parceria com os governos locais $5,44,46$. 
Figura 3

Linha do tempo com as principais medidas em resposta à pandemia de COVID-19. Coreia do Sul, 21 de janeiro de 2020 a 21 de janeiro de 2021.

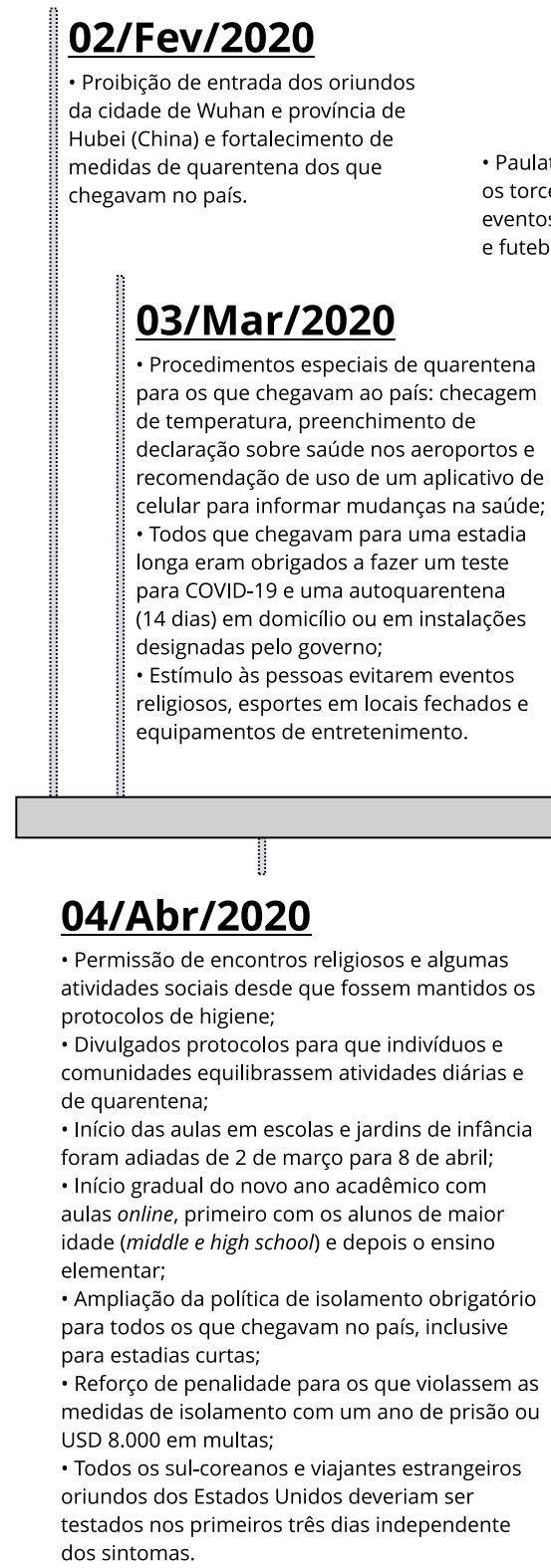

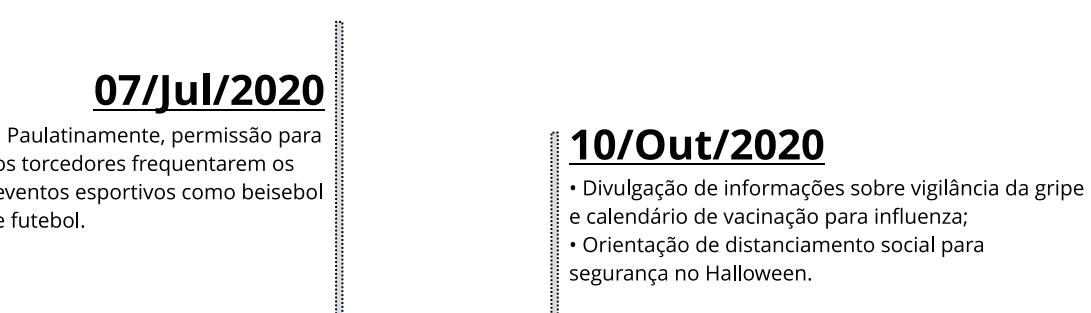

\section{5/Mai/2020}

\section{$11 /$ Nov/2020}

- Disposição das medidas de distanciamento dos níveis 1 a 3 ;

- Elevação dos níveis de distanciamento social para 1,5m, em Seul, na província de Gyeonggi e na cidade de Icheon;

- Elevação do nível de distanciamento social para $2 \mathrm{~m}$, nas áreas de Seul, Incheon, Gyeongg e nível 1,5m para Honam, região de Gwangju, Jeonbuk e Jeonnam.

- Recomendações para o feriado da

Colheita no outono.

- Início da temporada dos jogos de basquete profissional sem público: - Encerradas as seis semanas de campanha de distanciamento social forte com mudança para o chamado esquema de quarentena da vida cotidiana; - De 20 de maio a 8 de junho - após o país liberar o distanciamento social, em 6 de maio, as escolas abriram gradualmente, no entanto, as universidades, que em 9 de março iniciaram com aulas online, estenderam essa modalidade de ensino até o fim do semestre de primavera.

\section{8/Ago/2020}

- Medida de distanciamento social níve 2 é aplicada em todo o país; - Medida de distanciamento social "aprimorada" de nível 2 é aplicada para Seul, área metropolitana.

\section{2/Dez/2020}

- Nível de distanciamento social 1,5m, exceto na área metropolitana de Seul, que permanece $2 \mathrm{~m}$ - Revisão dos critérios para encerrar o isolamento para casos COVID-19;

- Distanciamento social nível 2,5m, para a área metropolitana de Seul;

- Distanciamento social nível $1 \mathrm{~m}$ - solicitação para a população quanto à vigilância;

- Emissão de medidas especiais de prevenção de infecções para as férias de inverno.

Fonte: Agência Sul-coreana de Prevenção e Controle de Doenças (http://www.kdca.go.kr/, acessado em 30/Abr/2021).

De forma pioneira, foram implementados centros de triagem na modalidade drive-thru, no intuito de ampliar os diagnósticos com redução da exposição dos trabalhadores de saúde 39,45,48. Os testes levavam em média 10 minutos para serem realizados e os resultados eram enviados diretamente para os pacientes por meio de mensagens de texto em até três dias. Em regiões com dificuldades para a circulação de veículos foram desenvolvidas cabines compactas móveis para realização de testes (walk-thru) 39,48. Os médicos de saúde pública, profissionais de saúde que atuam durante o período de 
serviço militar, foram destacados no processo 15 . Eles eram enviados rapidamente para os epicentros da COVID-19 e realizavam os testes em Centros de Triagem, aeroportos e hospitais.

Foi criada uma linha telefônica direta, com atendimento 24 horas para realização de triagem pelo médico da atenção primária, de modo a orientar a conduta e direcionar o caso 44 . Em caso de sintomas, eram ofertados exames gratuitos 48 . Essa política de atenção aos casos, que objetivava a detecção precoce, diminuindo o ritmo de novas infecções, permitiu uma contenção mais efetiva da doença em comparação a outros países 5,39. Foram realizadas extensivas investigações epidemiológicas de campo, utilizando-se tecnologias de informação e comunicação (TIC), com cooperação intergovernamental 15 que, além de entrevistas e registros médicos dos pacientes, empregavam para identificar casos e contatos de movimentação de transações com cartões de crédito, GPS e circuitos de televisão 5,28,49. Após o surto de MERS, em 2015, um regulamento nacional facilitou o compartilhamento dessas investigações para o público em geral 5,39,50,51.

A investigação epidemiológica em larga escala foi possível devido à presença de 30 equipes de resposta rápida do KCDC e a ampliação do número de trabalhadores dos serviços locais 49 . Em aproximadamente 250 distritos uma força de trabalho temporária de oficiais do serviço de inteligência epidemiológica foi estabelecida com funcionários de nível elementar e médio de centros de saúde governamentais. Apesar desses trabalhadores não serem servidores regulares e qualificados, foram treinados em protocolos padronizados para o rastreamento de contatos. Vale registrar que, a partir de 1999, por meio do Programa de Treinamento em Epidemiologia de Campo, o país já adotava oficiais dos serviços de inteligência epidemiológica dos níveis central e provinciais na condução das investigações epidemiológicas 52 .

A conjunção de medidas de vigilância, de rastreamento de contatos e de ampliação e qualificação da força de trabalho, visava informar a população e produzir informações para apoiar as decisões das autoridades do país. Houve uso de dados e inteligência artificial (IA) para tornar ágil a resposta e ampliar a efetividade na coordenação das atividades 16 . O fato de a Coreia do Sul ter a mais rápida velocidade de Internet do mundo favoreceu o processo 19 . Aplicativos de celular e mapas online, desenvolvidos pela iniciativa privada e órgãos públicos, tiveram papel fundamental no monitoramento da evolução da epidemia, para alertar o público do risco de exposição e na rápida adaptação dos centros de testagem de acordo com as necessidades 48 .

Com o aumento dos casos e a dificuldade de rastreamento dos contatos de forma manual, o Ministério da Terra, Infraestrutura e Transporte desenvolveu um sistema integrado de vigilância para realizar essa atividade de forma automática, em colaboração com o KCDC e o Ministério da Ciência e Tecnologia 39,53. Adaptou-se uma plataforma de dados para cidades inteligentes já existente, combinando-se dados da Agência Nacional de Polícia, três empresas de telecomunicação 5 e 22 empresas de cartão de crédito, culminando no Sistema de Gerenciamento Inteligente da COVID-19 39, um sistema de suporte para investigação epidemiológica, que reduziu o prazo de rastreamento para menos de 10 minutos.

O MoHW ainda desenvolveu um aplicativo de celular para monitorar pacientes potenciais, com ameaças de penalidades legais (multas e prisões) para reforçar a limitação de movimento. Também o Ministério do Interior e Segurança disponibilizou um aplicativo para monitorar a autoquarentena e avaliar as condições de saúde dos contatos. Com o uso ampliado das tecnologias digitais, a questão entre o direito à privacidade e o uso de dados na resposta a problemas de saúde pública foi problematizada 54 . Críticas apontaram o excessivo uso do aparelho estatal para controle dos indivíduos por meio de técnicas digitais 55 , com violação das liberdades civis dos pacientes infectados e possibilitando estigma pela sociedade 56,57. Embora intervenções psicológicas e voltadas para a saúde mental tenham sido constatadas, foi apontada a necessidade de melhor abordagem pública para evitar colapso das estruturas de apoio social e a estigmatização dos pacientes 58 .

Mesmo não tendo sido fechadas as fronteiras e nem tendo sido imposto lockdown nacional 50 , observou-se uma diminuição de mobilidade em centros de transporte público. A quantidade de passageiros em todas as estações reduziu 40,6\% entre janeiro e março. Foi constatada mudança no número de passageiros no metrô de Seul em distintos momentos da epidemia de COVID-19 59. Os autores apontaram redução maior do fluxo de usuários nas estações de trabalho quando comparada a estações para lazer ${ }^{59}$. Um estudo sobre o uso do sistema público de compartilhamento de bicicletas 
mostrou maior utilização deste tipo de transporte pessoal e abandono voluntário dos transportes públicos convencionais 60 .

A partir de julho de 2020, quando o número de novos casos voltou a aumentar, foram publicados e ativados distintos níveis de medidas de distanciamento físico. Muitos casos confirmados apresentavam um histórico de viagens para diferentes países. Estados Unidos e Europa representavam 65,2\% destes 53. Em 31 de julho 61, dentre outras medidas, foi permitida apenas 10\% de ocupação nos estádios para as ligas de futebol e beisebol, com a compra dos ingressos online, uso obrigatório de máscaras nos trajetos e durante os eventos esportivos (Quadro 2).

Um estudo atribuiu à ocorrência de grande manifestação antigovernamental e ao relaxamento das políticas de distanciamento o aumento da incidência de casos em agosto de 20223 . O nível 2 de alerta foi ativado a partir de 15 de agosto de 2020 62,63, devido aos clusters locais e transmissões comunitárias na área metropolitana de Seul e outras províncias. Em 30 de setembro, o número de novos casos reduziu novamente e houve diminuição do nível de distanciamento para o nível $1 \mathrm{em}$ outubro de 2020 com aumento de 1,5 a 2,5 em novembro e meses subsequentes, devido ao crescimento dos casos de transmissão local 64 (Tabela 1; Quadro 2; Figura 2). No mês de fevereiro de 2021, constatou-se decréscimo dos casos com mortalidade de 29,78 mortes/milhão de habitantes, enquanto observavam-se taxas muito maiores no Reino Unido (1.732,64 mortes/milhão), Itália (1.551,97 mortes/milhão), Portugal (1.511,37 mortes/milhão), Estados Unidos (1.439,01 mortes/milhão) e Brasil (1.128,03 mortes/milhão) 65 .

Em fevereiro de 2021, a KDCA noticiou o início da imunização no país para a COVID-19 66. As primeiras doses das vacinas Pfizer-BioNTech, adquiridas por meio da COVAX Facility, foram destinadas aos funcionários dos hospitais de referência para COVID-19. O país diversificou a compra de imunizantes destinada à vacinação de pessoas lotadas em centros de saúde e hospitais de recuperação. Cadastros eletrônicos foram realizados para a identificação dos elegíveis, segundo os critérios definidos. Não foram identificadas publicações que analisassem o porquê deste país ter iniciado a vacinação apenas no início de 2021.

A Coreia do Sul adotou um conjunto de medidas compensatórias e de proteção social, visando diminuir o impacto econômico da COVID-19 e a garantia da manutenção da vida dos cidadãos 65 . O governo anunciou o orçamento das medidas de mitigação da pandemia e de seus efeitos econômicos, com aprovação de um conjunto de medidas fiscais que incluíam garantia de créditos, suporte emergencial para famílias, empresas de turismo e indústria de exportação.

Houve subsídios de emergência sob forma de auxílios oferecidos na forma de cupons e dinheiro para estimular o consumo privado. Destacam-se, ainda, o aporte orçamentário com foco em criação de empregos; empréstimos de emergência para empresas em dificuldades; o Projeto Korean New Deal, no qual o país planejou criar 1,9 milhão de novos empregos e investir 133 bilhões de dólares, até 2025, com foco em projetos digitais e projetos “ecoamigáveis”. No âmbito do trabalho, buscaram-se medidas de modo a reduzir a exposição dos trabalhadores à COVID-19 no local de trabalho, com publicação de protocolo e estímulo a arranjos flexíveis (home office, horários flexíveis, entre outras medidas). Foram estabelecidos suporte de renda para trabalhadores doentes e suas famílias, para pessoas que perderam seus trabalhos ou rendas de trabalhos autônomos, estímulo às empresas para ajustar os horários de trabalho e preservar os empregos e suporte financeiro àquelas com redução na demanda, assim como a suspensão de pagamentos de tarifas, incluindo contas de eletricidade.

\section{Discussão}

O estudo realizado trouxe evidências que permitem relacionar o sucesso da Coreia do Sul no controle da pandemia COVID-19 a uma combinação de estratégias que incluem as práticas de vigilância, a investigação oportuna dos casos, o uso de tecnologias digitais e IA, além de outras de escopo intersetorial. Além disso, a coordenação nacional, exercida por órgão colegiado, qualificada tecnicamente e centralizada em um órgão vinculado ao MoHW, foi apontada como um dos fatores positivos para a resposta à COVID-19 7,19. 
Dessa forma, a resposta apoiada em dados epidemiológicos orientou a adoção de medidas e o acionamento tempestivo do protocolo de resposta às doenças infecciosas. A implementação de ações de vigilância adequadas permitiu um distanciamento mais flexível de acordo com a situação epidemiológica local.

Também merece destaque a avaliação dos casos suspeitos em Centros Locais de Triagem, com encaminhamento para unidades de acordo com o grau de gravidade. Esses achados são reforçados por estudos 5,8 que consideram como uma das principais lições aprendidas da experiência da Coreia do Sul o reconhecimento precoce da ameaça, com rápida ativação do protocolo nacional de emergência por liderança nacional e desencadeamento do processo para o pronto estabelecimento da capacidade diagnóstica do país.

A literatura aponta que a resposta negativa à MERS, em 2015, estimulou o governo sul-coreano a elaborar protocolo, incluindo a constituição de parcerias público-privadas, sob a regulação do Estado, para o desenvolvimento de testes de alta sensibilidade 16,44,47,51,67. A referida experiência negativa teria induzido a produção de tecnologias a exemplo da implantação de clínicas drive-thru e a cultura do uso de máscaras cirúrgicas no país 19.

Por outro lado, a epidemia de SARS, em 2004, levou ao KCDC a reformular o sistema de preparação e resposta para doenças infecciosas importadas, por meio da implantação de um Centro de Operação de Emergências, fortalecimento da comunicação de risco e da estratégia de avaliação de risco, com a criação de novas divisões e ampliação do número de profissionais para investigação epidemiológica 68.

Outros trabalhos afirmam que houve preocupação com perdas econômicas substantivas, além do medo na população com uma possível nova experiência favorecer a adesão às orientações do governo 5,34 .

As medidas de contenção após os primeiros casos, com uma rigorosa política de testagem para a identificação precoce destes, rastreamento de contatos, com recomendação de quarentena ou isolamento de forma oportuna foram fundamentais. Esses achados são ratificados por estudos comparativos $9,10,11$.

Destaque-se que as TIC foram elencadas como elemento importante na resposta à pandemia, assim como a disponibilização dos resultados dos testes e informações mais recentes sobre a COVID-19 nos sites governamentais e locais; as estratégias de comunicação integradas dos governos, incluindo os municípios; a estação de teste walk-thru. A interação intragovernamental e a resposta intersetorial parecem ter sido elementos também relevantes. Uma investigação apontou que os países que estiveram mais bem equipados e melhor preparados para ampliar a testagem e infraestrutura hospitalar, para rastrear contatos, entre outras medidas, responderam melhor à epidemia 39. Esses resultados são reforçados por outros autores 14 , que apontam a construção de painéis digitais epidemiológicos em tempo real, mapas interativos para localização de casos, uso de aplicativos móveis e georeferenciamento para monitorar o cumprimento da quarentena como fatores que contribuíram no enfrentamento da pandemia.

Embora os resultados desta revisão caracterizem a atenção primária na Coreia do Sul como distante de uma estrutura em rede, resolutiva e coordenadora do cuidado, aproximando-se de uma atenção primária seletiva 69 , a organização da resposta à pandemia utilizou a sua estrutura tanto em relação à infraestrutura física e humana, como ainda a constituição das parcerias locais entre diferentes serviços parece ter sido elemento facilitador na recepção dos usuários sintomáticos respiratórios para o encaminhamento e gestão do caso.

A importância da atenção primária no controle do enfrentamento da pandemia tem sido bem documentada 70,71,72, sendo particularmente efetiva nos casos leves e moderados, na coordenação do usuário na rede, assim como no monitoramento clínico e do isolamento, além do acompanhamento dos mesmos pós-COVID-19 70,73,74. A ampliação das estruturas existentes com a articulação das mesmas à atenção primária permitiu o controle da doença e evitou a sua propagação.

Além disso, vale destacar que o sistema de saúde sul-coreano se apresenta substantivamente privado no que se refere a prestação de serviços e à cobertura assistencial da maioria da população. Entretanto, a mobilização das estruturas públicas disponíveis nas instâncias provinciais e locais foi fundamental para a resposta à COVID-19. Nos casos em que houve necessidade de mobilização de 
estruturas não públicas a ação governamental foi determinante na regulação dos serviços, como no controle da situação em Daegu, emitindo suporte jurídico legal para as intervenções necessárias 31 .

Considerando o diagrama da vigilância da saúde adotado em estudos no Brasil 75, observa-se pelos resultados desta revisão que na Coreia do Sul foram desenvolvidas intervenções para o controle de risco e danos, além de adotadas medidas para prevenir os efeitos econômicos e sociais da pandemia, com estratégias de comunicação de risco capazes de promover o engajamento da população, dos profissionais, gestores e técnicos envolvidos.

\section{Considerações finais}

As evidências produzidas com base na revisão integrativa realizada apontam que a Coreia do Sul conseguiu controlar a transmissão e mitigar os danos da pandemia, com achatamento da curva epidêmica e sem colapso dos serviços de saúde, sugerindo que esse país corresponde a um caso de sucesso na resposta à COVID-19.

Houve articulação na resposta sul-coreana entre a vigilância, controle de riscos e danos e atuação sobre certos determinantes sociais para mitigar os efeitos socioeconômicos da pandemia. O país apresenta especificidades, como ter vivenciado a epidemia de MERS, que resultou na reforma institucional do sistema de enfrentamento às doenças infecciosas, na criação de protocolo com distintos níveis de intervenção em situação de epidemia e na disponibilidade de legislação sobre o uso de dados privados em situação de emergências sanitárias, permitindo maior adesão da população às ferramentas empregadas, além da disponibilização de arcabouço jurídico normativo para sustentar as ações do governo no enfrentamento da epidemia.

Os resultados do presente estudo permitem identificar um conjunto de lições com base na experiência sul-coreana visando o controle e manejo da doença. Destaca-se a importância de uma coordenação nacional expressiva, articulando as ações dos diversos ministérios e níveis de governo, com orientação para um trabalho intersetorial. A decisão governamental coordenada foi baseada em informação epidemiológica, com adoção de medidas de suporte e destinação de recursos para o enfrentamento da epidemia e de seus efeitos na economia do país. Houve a disponibilidade de protocolos em cada localidade contendo níveis de alerta para ativação de medidas a depender da situação epidemiológica, assim como comunicação de risco adequada utilizando-se ferramentas e tecnologias diversas, com atualização diária da situação e divulgação ampla para a sociedade, além do envio diário de mensagens de alerta, evidenciando áreas de maior risco. A vigilância de fronteiras foi realizada mesmo antes da confirmação do primeiro caso.

Foram utilizadas tecnologias digitais (aplicativos para dispositivos móveis) como ferramenta para vigilância de casos, monitoramento da autoquarentena e da situação de saúde de casos e contatos, ou para acompanhar a mobilidade no país. Foi ampliada a capacidade laboratorial e foram implantados centros de testagem favorecendo uma estratégia que possibilitasse um rastreamento de contatos em larga escala, com detecção precoce de casos, adoção de isolamento ou quarentenas de forma oportuna, levando a identificação de clusters e adoção de medidas de controle da transmissão.

Estruturas provisórias com ampliação de leitos foram criadas com vistas a atender os casos de menor gravidade e a articulação com a atenção primária ocorreu para diagnóstico, encaminhamentos e parcerias locais para acolhimento dos sintomáticos respiratórios. Destaque-se a importância da integração da atenção primária na resposta à pandemia e a garantia da disponibilidade de equipamento de proteção individual para os trabalhadores da saúde nos diversos níveis de atenção. Ademais, muitos trabalhadores foram convocados para a atividade de investigação epidemiológica e rastreamento de contatos nos serviços locais, o que possibilitou ampliar as ações da vigilância epidemiológica.

Cumpre destacar certas limitações do presente estudo, como em qualquer estudo de revisão, entre as quais ter como uma das fontes de produção dos dados artigos publicados que apresentam diversidade na construção das perguntas de investigação, delineamento metodológico e na análise. Assim, sugere-se o aprofundamento por meio de estudos comparativos, dispondo de maior rigor metodológico com uniformidade na produção e análise dos dados, com vistas a possibilitar uma melhor comparação entre os países. 
A construção de uma resposta efetiva na Coreia do Sul, centrada na informação epidemiológica, orientou a adoção de medidas para ampliação da infraestrutura física e profissional, da capacidade de resposta laboratorial, de atenção à saúde e de vigilância e monitoramento de casos e contatos. Portanto, seu sucesso, deve ser atribuído à conjunção de estratégias de diferentes naturezas, destacando-se as ações de coordenação nacional articuladas com os níveis locais, a vigilância e investigação dos casos.

\section{Colaboradores}

T. R. A. Rossi, C. L. M. Soares e G. A. Silva contribuíram com o planejamento do estudo, produção e análise dos dados, escrita e revisão. J. S. Paim contribuiu com a análise dos dados, escrita e revisão. L. M. Vieira-da-Silva contribuiu com o planejamento do estudo, análise dos dados, escrita e revisão.

\section{Informações adicionais}

ORCID: Thais Regis Aranha Rossi (0000-00022561-088X); Catharina Leite Matos Soares (00000002-8131-4831); Gerluce Alves Silva (0000-00020978-3393); Jairnilson Silva Paim (0000-00030783-262X); Lígia Maria Vieira-da-Silva (00000003-2518-411X).

\section{Agradecimentos}

Ao Conselho Nacional de Desenvolvimento Científico e Tecnológico e ao Ministério da Saúde (Chamada MCTIC/CNPQ/FNDCT/MS/SCTIE/ DECIT - 07/2020).

\section{Referências}

1. Barreto ML, Barros AJD, Carvalho MS, Codeço CT, Hallal PRC, Medronho RA, et al. O que é urgente e necessário para subsidiar as políticas de enfrentamento da pandemia de COVID-19 no Brasil? Rev Bras Epidemiol 2020; 23:e200032.

2. Greer S, de Ruijter A. EU health law and policy in and after the COVID-19 crisis. Eur J Public Health 2020; 30:623-4.

3. Sohrabi C, Alsafi Z, O’Neill N, Khan M, Kerwan A, Al-Jabir A, et al. World Health Organization declares global emergency: a review of the 2019 novel coronavirus (COVID-19). Int J Surg 2020; 76:71-6.

4. Singer DRJ. Healthcare impact of COVID-19 epidemic in India: a stochastic mathematical model. Med J Armed Forces India 2020; 76:147-55.

5. Oh J, Lee JK, Schwartz D, Ratcliffe HL, Markuns JF, Hirschhorn LR. National response to COVID-19 in the Republic of Korea and lessons learned for other countries. Health Syst Reform 2020; 6:e1753464.

6. Hitchie H. Emerging COVID-19 success story: South Korea learned the lessons of MERS. https://ourworldindata.org/COVID-exem plar-south-korea (acessado em 16/Fev/2021).

7. Tanne JH, Hayasaki E, Zastrow M, Pulla P, Smith P, Rada AG. COVID-19: how doctors and healthcare systems are tackling coronavirus worldwide. BMJ 2020; 368:m1090.

8. Yoon YK, Lee J, Kim SI, Peck KR. A systematic narrative review of comprehensive preparedness strategies of healthcare resources for a large resurgence of COVID-19 nationally, with local or regional epidemics: present era and beyond. J Korean Med Sci 2020; 35:e387. 
9. Chen H, Shi L, Zhang Y, Wang X, Sun G. A cross-country core strategy comparison in China, Japan, Singapore and South Korea during the early COVID-19 pandemic. Global Health 2021; 17:22.

10. Wang Z, Duan Y, Jin Y, Zheng Z. Coronavirus disease 2019 (COVID-19) pandemic: how countries should build more resilient health systems for preparedness and response. Glob Health J 2020; 4:139-45.

11. Lee SM, Lee D. Lessons learned from battling COVID-19: the Korean experience. Int J Environ Res Public Health 2020; 17:7548.

12. Park J. Institutions matter in fighting COVID-19: public health, social policies, and the control tower in South Korea. In: Greer SL, King EJ, Peralta-Santos A, Fonseca EM, editores. Coronavirus politics: the comparative politics and policy of COVID-19. Ann Arbor: University of Michigan Press; 2021. p. 106-26.

13. Reis RF, Quintela BM, Campos JO, Gomes JM, Rocha BM, Lobosco M, et al. Characterization of the COVID-19 pandemic and the impact of uncertainties, mitigation strategies, and underreporting of cases in South Korea, Italy, and Brazil. Chaos Solitons Fractals 2020; 136:109888.

14. Nageshwaran G, Harris RC, Guerche-Seblain $\mathrm{CE}$. Review of the role of big data and digital technologies in controlling COVID-19 in Asia: public health interest vs. privacy. Digit Health 2021; 7:20552076211002953.

15. Choi S. A hidden key to COVID-19 management in Korea: public health doctors. J Prev Med Public Health 2020; 53:175-7.

16. You J. Lessons from South Korea's COVID-19 policy response. Am Rev Public Adm 2020; 50: 801-8.

17. Whittemore R, Knafl K. The integrative review: updated methodology. J Adv Nurs 2005; 52:546-53.

18. WHO Regional Office for the Western Pacific. Republic of Korea health system review. https://apps.who.int/iris/handle/ 10665/208215 (acessado em 03/Fev/2021).

19. Palaniappan A, Dave U, Gosine B. Comparing South Korea and Italy's healthcare systems and initiatives to combat COVID-19. Rev Panam Salud Pública 2020; 44:e53.

20. Jeong H-S. Korea's National Health Insurance: lessons from the past three decades. Health Aff (Millwood) 2011; 30:136-44.

21. Walker ME, Anonson J, Klotz K, Gallant S, McDougall A, Yarie D, et al. Primary health care in South Korea: does it exist? How can health educators and health practitioners improve it? Int J Health Promot Educ 2009; 47:128-35.

22. Korea Disease Control and Prevention Agency. Opening of Korea Disease Control and Prevention Agency. Press release. September 14, 2020. https://www.kdca.go.kr/board/board. es? $\mathrm{mid}=\mathrm{a} 30402000000 \& \mathrm{bid}=0030$ (acessado em 03/Ago/2020).
23. Jeong GH, Lee HJ, Lee J, Lee JY, Lee KH, Han YJ, et al. Effective control of COVID-19 in South Korea: cross-sectional study of epidemiological data. J Med Internet Res 2020; 22:e22103.

24. Ha KM. Emergency response to the outbreak of COVID-19: the Korean case. Microbes Infect 2020; 22:159-61.

25. Lee D, Heo K, Seo Y, Ahn H, Jung K, Lee S, et al. Flattening the curve on COVID-19: South Korea's measures in tackling initial outbreak of coronavirus. Am J Epidemiol 2020; 190:496-505.

26. Kim H. The sociopolitical context of the COVID-19 response in South Korea. BMJ Glob Health 2020; 5:e002714.

27. Kim I, Lee J, Lee J, Shin E, Chu C, Lee SK. KCDC risk assessments on the initial phase of the COVID-19 outbreak in Korea. Osong Public Health Res Perspect 2020; 11:67-73.

28. COVID-19 National Emergency Response Center, Epidemiology \& Case Management Team, Korea Centers for Disease Control \& Prevention. Contact transmission of COVID-19 in South Korea: novel investigation techniques for tracing contacts. Osong Public Health Res Perspect 2020; 11:60-3.

29. Shaw R, Kim Y, Hua J. Governance, technology and citizen behavior in pandemic: lessons from COVID-19 in East Asia. Prog Disaster Sci 2020; 6:100090.

30. Ki M; Task Force for 2019-nCoV. Epidemiologic characteristics of early cases with 2019 novel coronavirus (2019-nCoV) disease in Korea. Epidemiol Health 2020; 42:e2020007.

31. World Health Organization. WHO coronavirus (COVID-19) dashboard. https://COVID19. who.int/ (acessado em 20/Fev/2021).

32. Kim S, Castro MC. Spatiotemporal pattern of COVID-19 and government response in South Korea (as of May 31, 2020). Int J Infect Dis 2020; 98:328-33.

33. Shim E, Tariq A, Choi W, Lee Y, Chowell G. Transmission potential and severity of COVID-19 in South Korea. Int J Infect Dis 2020; 93:339-44.

34. Hwang J, Park H, Kim S-H, Jung J, Kim N. Basic and effective reproduction numbers of COVID-19 cases in South Korea excluding Sincheonji cases. medRxiv 2020; 31 mar. https://www.medrxiv.org/content/10.1101/2 020.03.19.20039347v2.

35. Dighe A, Cattarino L, Cuomo-Dannenburg G, Skarp J, Imai N, Bhatia S, et al. Response to COVID-19 in South Korea and implications for lifting stringent interventions. BMC Med 2020; 18:321.

36. Al-Rousan N, Al-Najjar H. Data analysis of coronavirus COVID-19 epidemic in South Korea based on recovered and death cases. J Med Virol 2020; [Epub ahead of print]. 
37. Ryu S, Ali ST, Lim J-S, Chun BC. Estimation of the excess COVID-19 cases in Seoul, South Korea by the students arriving from China. Int J Environ Res Public Health 2020; 17:3113.

38. Organisation for Economic Co-operation and Development. OECD country tracker policy COVID-19. https://www.oecd.org/coronavi rus/country-policy-tracker/ (acessado em 20/ Fev/2021).

39. Lee D, Lee J. Testing on the move: South Korea's rapid response to the COVID-19 pandemic. Transp Res Interdiscip Perspect 2020; 5:100111

40. Lee M, You M. Psychological and behavioral responses in South Korea during the early stages of coronavirus disease 2019 (COVID-19). Int J Environ Res Public Health 2020; 17:2977.

41. Yu S, Kim S, Kang J. Face mask policies in South Korea in response to COVID-19. Asia Pac J Public Health 2020; 32:497-9.

42. Joint statement of the Korean Society for Preventive Medicine and the Korean Society of Epidemiology on the response to the COVID-19 outbreak. Epidemiol Health 2020; 42:e2020019.

43. Petersen E, Wasserman S, Lee SS, Go U, Holmes AH, Al-Abri S, et al. COVID-19 - We urgently need to start developing an exit strategy. Int J Infect Dis 2020; 96:233-9.

44. Ku SS, Choe YJ. A public-private partnership model to build a triage system in response to a COVID-19 outbreak in Hanam City, South Korea. Osong Public Health Res Perspect 2020; 11:339-42.

45. Choi JY. COVID-19 in South Korea. Postgrad Med J 2020; 96:399-402.

46. Han E, Tan MMJ, Turk E, Sridhar D, Leung GM, Shibuya K, et al. Lessons learnt from easing COVID-19 restrictions: an analysis of countries and regions in Asia Pacific and Europe. Lancet 2020; 396:1525-34.

47. Kang J, Jang YY, Kim J, Han SH, Lee KR, Kim $\mathrm{M}$, et al. South Korea's responses to stop the COVID-19 pandemic. Am J Infect Control 2020; 48:1080-6.

48. Kim S, Lee JY. Walk-through screening center for COVID-19: an accessible and efficient screening system in a pandemic situation. J Korean Med Sci 2020; 35:e154.

49. Korea Centers for Disease Control and Prevention. Coronavirus disease-19, Republic of Korea. http://ncov.mohw.go.kr/en/ (acessado em 20/Fev/2021).

50. Lee D, Heo K, Seo Y. COVID-19 in South Korea: lessons for developing countries. World Dev 2020; 135:105057.

51. Lu N, Cheng KW, Qamar N, Huang KC, Johnson JA. Weathering COVID-19 storm: successful control measures of five Asian countries. Am J Infect Control 2020; 48:851-2.

52. Kwon G-Y, Moon S, Kwak W, Gwack J, Chu C, Youn S-K. Epidemic intelligence service officers and field epidemiology training program in Korea. Osong Public Health Res Perspect 2013; 4:215-21.
53. Arora AS, Rajput H, Changotra R. Current perspective of COVID-19 spread across South Korea: exploratory data analysis and containment of the pandemic. Environ Dev Sustain 2020; [Epub ahead of print].

54. Kim SW, Lee KS, Kim K, Lee JJ, Kim JY; Daegu Medical Association. A brief telephone severity scoring system and therapeutic living centers solved acute hospital-bed shortage during the COVID-19 outbreak in Daegu, Korea. J Korean Med Sci 2020; 35:e152.

55. Baca G. Eastern surveillance, Western malaise, and South Korea's COVID-19 response: oligarchic power in Hell Joseon. Dialect Anthropol 2020; 44:301-7.

56. Yi J, Lee W. Pandemic nationalism in South Korea. Society 2020; 57:446-51.

57. Park S, Choi GJ, Ko H. Information technology-based tracing strategy in response to COVID-19 in South Korea: privacy controversies. JAMA 2020; 323:2129-30.

58. Jung SJ, Jun JY. Mental health and psychological intervention amid COVID-19 outbreak: perspectives from South Korea. Yonsei Med J 2020; 61:271-2.

59. Park J. Changes in subway ridership in response ta COVID-19 in Seoul, South Korea: implications for social distancing. Cureus 2020; 12:e7668.

60. Park S, Kim B, Lee J. Social distancing and outdoor physical activity during the COVID-19 outbreak in South Korea: implications for physical distancing strategies. Asia Pac J Public Health 2020; 32:360-2.

61. Korea Centers for Disease Control and Prevention. Press release. July 31, 2020. https://www.kdca.go.kr/board/board.es? $\operatorname{mid}=a 30402000000 \& b i d=0030$ (acessado em 03/Ago/2020).

62. Korea Centers for Disease Control and Prevention. Press release. August 15, 2020. https://www.kdca.go.kr/board/board.es? $\mathrm{mid}=\mathrm{a} 30402000000 \& \mathrm{bid}=0030$ (acessado em 15/Mar/2021).

63. Korea Centers for Disease Control and Prevention. Press release. August 30, 2020. https://www.kdca.go.kr/board/board. es? $\mathrm{mid}=$ a30402000000\&bid $=0030$ (acessado em 15/Mar/2021).

64. Korea Centers for Disease Control and Prevention. Press release. October 12, 2020. https://www.kdca.go.kr/board/board. es? $\mathrm{mid}=\mathrm{a} 30402000000 \& \mathrm{bid}=0030$ (acessado em 03/Mar/2021).

65. Our World in Data. Coronavirus pandemic (COVID-19). https://ourworldindata.org/ (acessado em 01/Mar/2021).

66. Korea Disease Control and Prevention Agency. COVID-19 vaccination to begin this week. Press release. February 22, 2021. https://www.kdca.go.kr/board/board. es? $\mathrm{mid}=\mathrm{a} 30402000000 \& \mathrm{bid}=0030$ (acessado em 03/Mar/2021). 
67. Park J, Chung E. Learning from past pandemic governance: early response and public-private partnerships in testing of COVID-19 in South Korea. World Dev 2021; 137:105198.

68. Cho H-W. Effectiveness for the response ta COVID-19: the MERS outbreak containment procedures. Osong Public Health Res Perspect 2020; 11:1-2.

69. Aquino R, Medina MG, Nunes CA, Sousa MF. A Estratégia Saúde da Família e o reordenamento do sistema de serviços de saúde. In: Paim JS, Almeida Filho N, organizadores. Saúde coletiva: teoria e prática. Rio de Janeiro: Medbooks; 2014. p. 353-71.

70. Barbosa SP, Silva AVFG. A prática da atenção primária à saúde no combate da COVID-19. APS em Revista 2020; 2:17-9.

71. Nedel F. Enfrentando a COVID-19: APS forte agora mais que nunca! APS em Revista 2020; 2:11-6.
72. Facchini L. COVID-19: nocaute do neoliberalismo? Será possível fortalecer os princípios históricos do SUS e da APS em meio à pandemia? APS em Revista 2020; 2:3-10.

73. Sarti TD, Lazarini WS, Fontenelle LF, Almeida APSC. Qual o papel da atenção primária à saúde diante da pandemia provocada pela COVID-19? Epidemiol Serv Saúde 2020; 29:e2020166.

74. Daumas RP, Azevedo e Silva G, Tasca R, Leite IC, Brasil P, Greco DB, et al. O papel da atenção primária na rede de atenção à saúde no Brasil: limites e possibilidades no enfrentamento da COVID-19. Cad Saúde Pública 2020; 36:e00104120.

75. Paim JS. A reforma sanitária e os modelos assistenciais. In: Rouquayrol MZ, organizador. Epidemiologia \& saúde. São Paulo: Medsi; 1994. p. 455-66. 


\section{Abstract}

The studies published on the South Korean response to COVID-19 point to different reasons for the country's success. No reviews were identified on South Korea from January 2020 to April 2021 or that analyzed the pandemic's recrudescence. Aimed at better systematization of South Korea's success in controlling the epidemic, we conducted an integrative review to analyze that country's experience with the COVID-19 pandemic, seeking to identify the relationship between the measures adopted, the health system's characteristics, and evolution of the selected indicators. Various databases were used, beside epidemiological bulletins and press conferences of the Korea Centers for Disease Control and Prevention (KCDC). We also analyzed reports by the World Health Organization (WHO) and the European Observatory on Health Systems and Policies. The study's results allow identifying a set of lessons based on the South Korean experience with control and management of the disease. The response by South Korea was successful, due to action in the control of risks and harms, action on social determinants to mitigate the socioeconomic effects of the health crisis, prior experience with other respiratory disease epidem$i c s$, and effective national coordination.

Pandemics; COVID-19; Health Systems; Surveillance; Health Policy

\section{Resumen}

Los estudios publicados sobre la respuesta de Corea del Sur a la COVID-19 apuntan distintos motivos para su éxito. No se identificaron revisiones sobre Corea del Sur entre enero de 2020 y abril de 2021 o que analizaran el recrudecimiento de la pandemia. Con el fin de una mejor sistematización sobre el éxito en el control de la epidemia, se desarrolló una revisión integradora para analizar la experiencia de aquel país en el combate de la pandemia de COVID-19, buscando identificar la relación entre las medidas adoptadas, las características del sistema de salud y la evolución de indicadores seleccionados. Se utilizaron distintas bases de datos, además de los boletines epidemiológicos $y$ conferencias de prensa del Centro Surcoreano para el Control y la Prevención de Enfermedades $(K C D C)$. Asimismo, se analizaron informes de la Organización Mundial de la Salud (OMS) y del Observatorio Europeo de Politicas y Sistemas Sanitarios. Los resultados del presente estudio permiten identificar un conjunto de lecciones, basadas en la experiencia surcoreana, con el fin del control $y$ manejo de la enfermedad. La respuesta de Corea del Sur fue exitosa, debido a las acciones en el control de riesgos y daños, actuación sobre determinantes sociales para mitigar los efectos socioeconómicos de la crisis sanitaria, así como su experiencia previa en otras epidemias respiratorias $y$ su significativa coordinación nacional.

Pandemias; COVID-19; Sistemas de Salud; Vigilancia; Politica de Salud
Recebido em 18/Mai/2021

Versão final reapresentada em 05/Ago/2021 Aprovado em 19/Ago/2021 